\title{
Duality of singularities of multiscale damage localization and crack advance: length variety in Theory of Critical Distances
}

\author{
Oleg Naimark \\ Institute of Continuous Media Mechanics UB RAS, Russia \\ naimark@icmm.ru
}

\begin{abstract}
The existence of two singularities related to the stress field at the crack tip and blow-up kinetics of damage localization is considered as the physical basis for the interpretation of the Theory of Critical Distances. The free energy metastability of solid with defects and corresponding free energy release explain the conception of the Finite Fracture Mechanics in the presence of the finite amplitude energy barrier. The variety of crack paths is analyzed as duality of inherently linked two types of singularities related to the singularity of multiscale damage kinetics under crack nucleation and singularity of stress field at the crack tip as the classical framework of fracture mechanics. The singularity of multiscale damage kinetics is a natural precursor of crack nucleation that could provide in some cases the totally independent scenario of fracture from the stress singularity at the crack tips. The influence of two singularities with the nature of intermediate asymptotical solutions for stress at the crack tip and damage localization kinetics over the set of spatial scales represents two attractors, which provides the variety of crack paths for corresponding loading conditions.
\end{abstract}

KEYwORDS. Singularity duality; Critical distance theory.

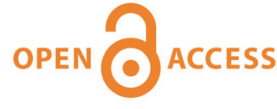

Citation: Naimark, O., Duality of singularities of multiscale damage localization and crack advance: length variety in Theory of Critical Distances, 49 (2019) 272-281.

Received: 12.04 .2019

Accepted: 29.05.2019

Published: 01.07.2019

Copyright: (C) 2019 This is an open access article under the terms of the CC-BY 4.0, which permits unrestricted use, distribution, and reproduction in any medium, provided the original author and source are credited.

\section{INTRODUCTION}

$\mathrm{N}$ onlinear aspects of multiscale damage evolution in the presence of stress concentration, cracks and notches are the subject of intensive experimental and theoretical studies in the problem of damage-failure transition for quasi-static, fatigue and dynamic statements, which generally are inherently linked. The phenomenological statements of this problem was formulated by the Theory of Critical Distances (TCD), which combines the approaches based on conventional material toughness and material strength parameters for the definition of material characteristic lengths [1]. However, there is a permanent question concerning physical basis for the TCD and the definition of characteristic lengths. The material length scales are the key parameters of physically based theories of fracture. These lengths correlate with microstructure scales (grain or inclusion sizes, spacing) and responsible for the susceptibility of material to multiscale damage accumulation [2]. 
The TCD had independent inventions in different forms for the prediction of quasi-brittle fracture [3, 4]. The theory of critical distances operates with the phenomenological estimation of the process zone $L$ arising from the continuum mechanics models. Commonly used length scale $L$ in the TCD is given by

$$
L=\frac{1}{\pi}\left(\frac{K_{c}}{\sigma_{0}}\right)^{2},
$$

where $K_{c}$ is the fracture toughness of the material, and $\sigma_{0}$ is material tensile strength. In fatigue problems the same equation has used, replacing these material constants with the relevant cyclic ones: the crack propagation threshold $\Delta K_{t h}$ and fatigue limit $\Delta \sigma_{0}$. Estimated by (1) the values of $\mathrm{L}$ for fracture and fatigue of brittle and ductile materials (silicon carbide and alumina [5], steel [6,7], fiber composite laminate [4]) separate the "damage induced" and "crack induced" fracture scenario: for small cracks the experimental data deviate from the Linear Fracture Mechanics (LFM) line towards tensile strength $\sigma_{0}$. Scale of $L$ occurs in the middle of transient region for "Fracture stress" versus "Defect size" curve [1].The value of $L$ determines structure-induced damage and fracture processes and are related to a microstructural parameter, for example, the grain size for ceramic materials. For metal fatigue $L$ is associated with the length of nonpropagating cracks [8]. Critical Distance approach is generalization of the Griffith energy balance theory assuming a finite amount of crack extension, that is so-called as the Finite Fracture Mechanics (FFM) [9,10]. This FFM can be formulated in the form:

$$
\int_{0}^{2 L} K^{2} d a=K_{c}^{2} \cdot 2 L .
$$

Three-point bending analysis showed the deviation both LFM and FFM for "normalized strength" $\sigma / \sigma_{0}$ versus "normalized beam height" $h / 2 L$ at the vicinity of $L$ : the LM and FFM tend to opposite extremes as the beam height approaches $L$. However, a combined approach, in which both LFM and FFM criteria apply, show realistic results supporting numerous structural observations revealing the variety of length $L$. Using this approach it was the prediction of the size effect in notched and un-notched beams [10] and the fracture criterion was proposed with application to laminate cracking analysis $[11,12]$.

Comparing experimental data on fracture strength as a function of crack length revealed the similarity in form for both monotonic fracture in brittle materials such as ceramics, and for fatigue limit behavior in metals. It is the cases, when Linear Fracture Mechanics breaks down at short-cracks scale, that are significant in predicting the material responses to small defects such as manufacturing flaws, and in estimating the number of cycles needed for small fatigue cracks to grow. The anomalous short-crack behavior highlights more general problem of size effects or scaling effects. The TCD is useful for the applications, when the size of the sample is approaching to $L[1,9,10]$.

It is important observation that the effect of notch-root radius has also interpretation in TCD phenomenology to introduce a critical root radius for crack-like behavior. This characterization of notch effects is based on the TCD phenomenology and has important applications for more complex effects related to multiaxial loading and finite life predictions $[2,8]$. The interpretation of variation in specimen strength when the size of the entire specimen is changed (saving the shape) is another complex problem addressing to TCD with applications to high cycle fatigue and the fracture of quasi-brittle materials necessitating a modified form of the approach in which $L$ becomes a variable quantity [13,14]. The power of TCD approach in predicting a wide range of phenomena in different materials, and capability of engineering applications stimulate the question - why does this theory work and what is the ranges [1]?

Theoretical basis of the TCD is the Finite Fracture Mechanics (FFM) formulation that is combined the criticality features related to two stress-based factors: strength and fracture toughness [9]. Understanding of the scientific basis of the TCD includes the study of the nature of cohesive (process) zone, applications of local and non-local approaches. There is the question concerning the range of validity of the TCD since FFM formulation shares common features of singularities with LFM. It means, that the underlying scaling phenomena are related to the smallscale yielding criterion. The smallscale yielding criterion, for instance, for the notch-root plastic zone assumes a small fraction of specimen dimensions providing crack instability. The structural and mechanical basis of FMM and the "critical distances" conception is the existence of characteristic length providing the discontinuous and branching scenario of crack growth on the distances determined by 
the microstructure and deformation behavior of the material. The TCD provides two new material parameters: the distance $\mathrm{L}$ and the stress ratio $\sigma_{0} / \sigma_{u}$, where $\sigma_{u}$ is the tensile stress, $\sigma_{u}$ is, the so-called, characteristic strength of materials. These parameters are recognized as potential characteristics of a material's brittleness, notch sensitivity and susceptibility to size effects. The TCD is recognized as the approach providing a bridge between continuum-mechanics and micromechanical models, stress-based methods and stress-intensity methods, fatigue failure and brittle fracture with application to different classes of materials.

\section{SOME RESULTS IN CRACK MECHANICS AND FAILURE}

he subject of variety of crack paths is one whose roots go back to the classical work of Griffith [15]. With a goal to study the crack path problem and the interaction of the main crack with the defect ensemble in the process zone we will consider briefly the classical results in the crack mechanics, where much analytical progress has been made in assuming that the medium behaves according to the equations of linear elasticity. According to the Griffith theory the additional characteristics of the crack resistance were introduced in the form of the energy of the development of the new surface at the crack tip. The energy $U$ of elastic materials with a crack is represented in the form (Fig.1, curve 1)

$$
U=-\frac{\sigma^{2}}{2 E}\left(\frac{\pi a^{2}}{4}\right)+2 \gamma a,
$$

where $\gamma$ is the surface energy; $\sigma$ is the applied stress; $a$ is the crack length; $E$ is the elastic modulus.

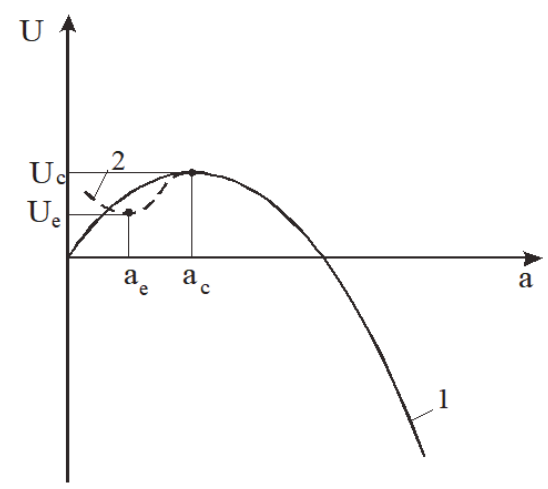

Figure 1: The Griffith (1) and Fraenkel (2) energy form of elastic solid with a crack.

Irwin [16] developed the Griffith conception and proposed the force version of the crack stability (the stress intensity factor) related to the intermediate self-similar singular solution for the stress field at the crack tip area

$$
\sigma_{i k} \approx K_{I} r^{-\frac{1}{2}} f_{i j}(\theta) \quad K_{I}=\sigma \sqrt{\pi a},
$$

where $K_{I}$ is the stress intensity factor, $r, \theta$ are the coordinates of point, $f_{i j}(\theta)$ is the $\theta$ dependence in the first term of asymptotical solution. Two classical treatments are based on the Griffith criterion and stress-intensity factor criterion and reflect the physical contradiction related to incorrectly prediction of an infinite load at failure due to the global instability (singularity) of stress field at the crack tip.

Barenblatt [17] proposed a variant of the force version of the crack stability that reflects another view on the role of stress in the crack tip area. It was assumed the existence of the cohesion forces $G_{B}(s)$ at the process (cohesive) area with the size $d \quad(0<s<d)$. The self-similarity of the crack tip evolution are the consequence of the small ratio of the applied stress $\sigma$ and the cohesion force $G_{B}: \sigma / G_{B}<<1$. This fact reflects the intermediate-asymptotic character of quasi-brittle failure theories and the material parameter was introduced, the so-called the cohesive modulus, as the independent strength property of materials: $K_{B}=G_{B} \sqrt{d}$. Despite the similar form of the cohesive modulus $K_{B}$ and the stress intensity 
factor $K_{I}$ there is a difference between the Irwin criteria and the cohesive modulus. The cohesive modulus determines the steady-state character of crack propagation, but not the catastrophic one corresponding to the Griffith-Irwin approach.

Non-local formulation of stress criterion was proposed by Novozhilov [3] to introduce the fracture "quantization" scale. According to this formulation the crack propagation condition in Mode I reads:

$$
\int_{0}^{d} \sigma_{y y} d x \geq 2 d \sigma_{C}
$$

where $\sigma_{y y}$ is the normal stress perpendicular to the atomic layer with the space $d, \sigma_{C}$ is the critical stress. The space $d$ is the fracture quantum. The criterion (5) can be used if the complete expression of the stress-field, and not only its asymptotic term, is taken into consideration. The non-local stress criterion can be also used for the interpretation of the cohesion forces $G_{B}(s)$, when the fracture quantum not restricted to be the atomic spacing.

The qualitative difference between above mentioned approaches can be shown taking in view the remarks by Fraenkel [18] under the critical analysis of the Griffith approach. Fraenkel wrote that the physically realistic form of the energy $U$ must contain the local minimum $U_{e}\left(a_{e}\right)$ (Fig. 1, curve 2). The difference in the energy $\Delta U=U_{c}-U_{e}$ determines the work of the stress field at the crack tip under transition from the steady-state to the unstable regime of crack propagation. This work provides the overcoming of the energy barrier. It is natural to assume that the cohesive modulus, non-local approach, the Finite Fracture Mechanics and the TCD are the force version of this energy barrier. We will show in the following that the metastable energy form, assumed by Fraenkel, has the relationship to the collective behavior of the defect ensemble in the process zone and to the interaction of the defects ensemble with the main crack.

\section{ORIGIN OF CHARACTERISTIC LENGTHS AND CRACK VARIETY PATHS}

$\mathrm{S}$ tatistical theory of typical mesoscopic defects (microcracks, microshears) allowed us to establish specific type of critical phenomena in solid with defects - the structural-scaling transitions and propose the phenomenology of damage-failure transition [19]. One of the key results of the statistical approach and statistically based phenomenology are the establishment of two "order parameters" responsible for the structure evolution - the defect density tensor $p_{i k}$ (defect induced deformation) and the structural scaling parameter $\delta=\left(R / r_{0}\right)^{3}$, which represents the ratio of the spacing between defects $R$ and mean size of structural heterogeneity $r_{0}$, and characterizes the current susceptibility of material to the defects growth [20,21]. Statistically predicted non-equilibrium free energy $F$ represents generalization of the Ginzburg-Landau expansion in terms of mentioned order parameters, defect induced deformation $p(x)=p_{y y}$ in uni-axial loading in $y$-direction and structural scaling parameter $\delta$ :

$$
F=1 / 2 A\left(\delta, \delta_{*}\right) p^{2}-1 / 4 B p^{4}-1 / 6 C\left(\delta, \delta_{c}\right) p^{6}-D \sigma p+\chi(\partial p / \partial x)^{2},
$$

where $\sigma=\sigma_{y y}$ is the stress, $\chi$ is the non-locality parameter, $A, B, C, D$ are the material parameters, $\delta_{*}$ and $\delta_{c}$ are characteristic values of structural-scaling parameter (bifurcation points) that define the areas of typical nonlinear material responses on the defect growth (quasi-brittle, ductile and fine-grain states) in corresponding ranges of $\delta$ : $\delta<\delta_{c} \approx 1, \delta_{c}<\delta<\delta_{*}, \delta>\delta_{*} \approx 1.3$. Free energy form (6) represents multi-wall potential with qualitative different metastability in the ranges $\delta_{c}<\delta<\delta_{*}$ and $\delta<\delta_{c} \approx 1$ [19].

Free energy release kinetics allows the presentation of damage evolution equation in the form

$\dot{p}=-\Gamma_{p}\left[A\left(\delta, \delta_{*}\right) p-B p^{3}-C\left(\delta, \delta_{c}\right) p^{5}-D \sigma\right]+\Gamma_{p} \partial / \partial x(\chi \partial p / \partial x)$ 
where $\Gamma_{p}$ are the kinetic coefficients. Kinetic equations (7) and the equation for the total deformation $\varepsilon=\hat{C} \sigma+p(\hat{C}$ is the component of the elastic compliance tensor) represent the constitutive equations of materials with defects. Material responses include the generation of characteristic collective modes - the autosolitary waves in the range of $\delta_{c}<\delta<\delta_{*}$ and the "blow-up" dissipative structure in the range $\delta<\delta_{c} \approx 1$. The generation of these collective modes under the loading provides the defect induced mechanisms of structural (ductile) relaxation in the range $\delta_{c}<\delta<\delta_{*}$ and specific mechanisms of damage localization on the set of spatial scales with the blow-up defect growth kinetics. The "blow-up" damage localization kinetics follows to the self-similar solution [21]

$$
p=g(t) f(\xi), \xi=x / L_{H}, g(t)=G\left(1-t / \tau_{c}\right)^{-m},
$$

and can be considered as the precursor of crack nucleation. The parameters in (8) are: $\tau_{c}$ is the so-called "peak time" $\left(p \rightarrow \infty\right.$ at $t \rightarrow \tau_{c}$ for the self-similar profile $f(\xi)$ of defects localized on the scale $\left.L_{H}\right), G>0, m>0$ are the parameters of non-linearity, which characterize the free energy release rate for $\delta<\delta_{c}$. The blow-up self-similar solution (8) describes damage kinetics for $t \rightarrow \tau_{c}, \quad p \rightarrow p_{c}$ (Fig.2) on the set of spatial scales $L_{H}=k L_{c}, \quad k=1,2, \ldots K$, where $L_{c}$ and $L_{H}$ corresponds to the so-called "simple" and "complex" blow-up dissipative structures. The scales $L_{c}$ represent natural measure of the quantization length in the process zone providing the variety of the crack paths in the presence of two singularities: intermediate asymptotic solution for stress distribution at the crack tip area and the blow-up damage localization kinetics in the process zone.

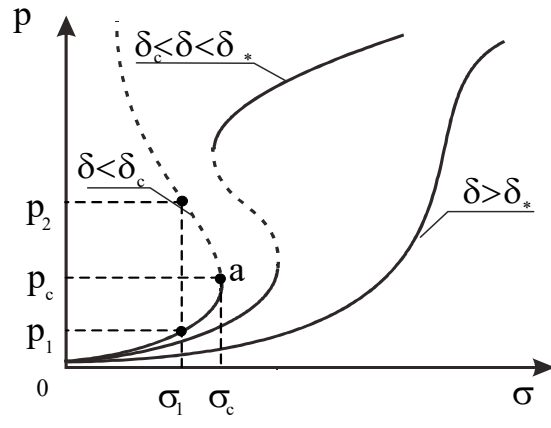

a

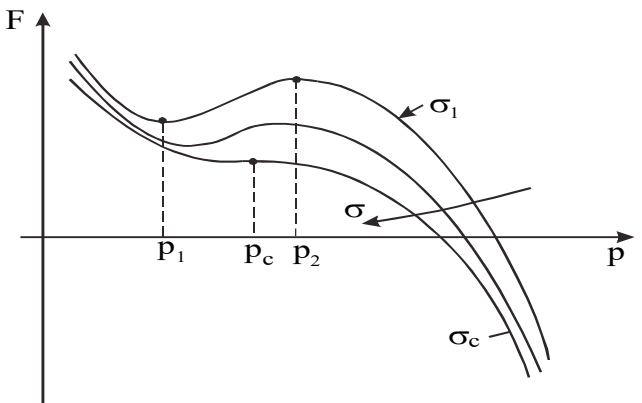

$\mathrm{b}$

Figure $2:$ a. Nonlinear responses of material on defect density $p$ in different ranges of structural-scaling parameter $\delta$; b. Free energy metastability for $\delta<\delta_{c} \approx 1$ [19].

\section{DUALITY OF SINGULARITIES. THE TCD LENGTHS}

7 he existence of two singularities related to the stress field at the crack tip (4) and blow-up kinetics of damage localization (8) represents the physical basis for the interpretation of phenomenological assumption of the Theory of Critical Distances. The free energy metastability of solid with defects and corresponding free energy release explain the conception of the Finite Fracture Mechanics in the presence of the finite amplitude energy barrier. The out-ofequilibrium system "solid with defects" realizes the transition in the areas of metastabilities $\left(\delta_{c}<\delta<\delta_{*}\right.$ and $\left.\delta<\delta_{c} \approx 1\right)$ due to the generation of collective modes of defects (autosolitary waves of strain localization transforming into the blowup dissipative structures), which provides finally singular damage kinetics on the set of spatial lengths $L_{H}=k L_{c}, \quad k=1,2, \ldots K$. Different nature of these singularities, related to spatial (geometrical) basis for stress intensity factor and temporal one for damage localization, leads to the variety of transition scenario to failure in wide range of load intensity. Experimental study of dynamic crack instability in PMMA revealing the transition from steady to branching regimes of crack dynamics with qualitative changes of fracture surface morphology [19, 20], Fig.3; the "resonance" excitation of selected blow-up modes (equal size of mirror-like damage localization zones in different spall cross-sections of shocked PMMA rod $[21,22])$, the limit case of resonance damage localization under the "failure wave" initiation [23, 24], temporal and spatial scaling under dynamic fragmentation [25] supported the competitive role of two mentioned 
singularities (attractor types) and allow the discussion of the area, when the TCD can be effectively used. The area of competition between two attractors belongs to the intermediate temporal scales of the loading rate, that is close to $t \rightarrow 10^{-6}-10^{-5} s[23]$.

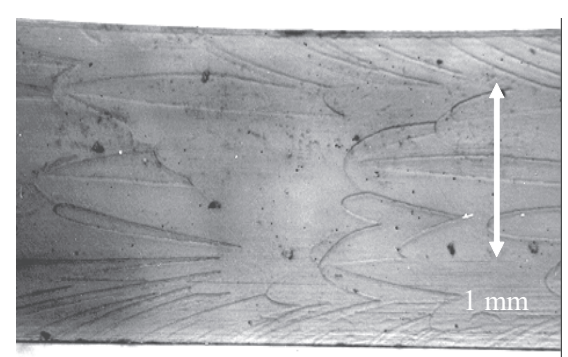

a

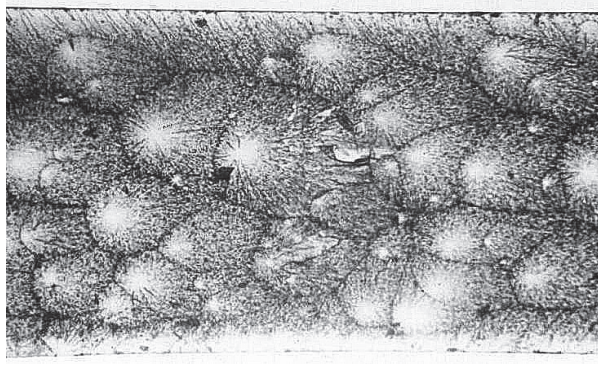

$\mathrm{b}$

Figure 3: Typical fractographic images of fracture surface for steady-state (a) and branching (b) regime of crack dynamics [24].

The TCD is based on the "force" version of failure criteria and, as a consequence, the large class of load conditions (with characteristic times approaching to the time of blow-up damage kinetics) could not be considered in the framework of the TCD phenomenology. Characteristic example for such situation is the transition from the steady-state to the branching crack dynamics in PMMA with qualitative changes of the fracture surface morphology: continuously mirror fracture surface for the steady-state crack dynamics and pronounced roughness with numerous mirror zones having different characteristic sizes (Fig.3). Steady-state crack dynamics and mirror morphology corresponds to the LFM asymptotical solution (4). The branching crack scenario corresponds to the set of collective modes of damage localization (the set of mirror zones), that represents the "degrees of freedom" responsible for the creation of the "process zone length" providing the crack advance. The formation of the "process zone length" is the consequence of the free energy metastability and "decomposition" of the metastable states due to the generation of collective blow-up defects modes corresponding to the self-similar solution (8) [26]. The existence of self-similar profile of damage distribution $f(\xi)$, localized on the set of scales $\left(L_{H}=k L_{c}, \quad k=1,2, \ldots K\right)$, explains the nature of the Critical Distances, that provides both scenario of damage-failure transition corresponding to stress-based and stress-intensity phenomenology. The selection of wide spectrum of scales in the range $L_{H}=k L_{c}, k=1,2, \ldots K$ is realized under dynamic and shock wave loading according to the presence of wide spectrum of finite amplitude stress modes and, as the consequence, corresponding spectrum of blow-up damage localization modes. Phenomenological basis of the DCT approach corresponds to the quasistatic loading conditions, when the "complex" blow-up mode can be initiated with the length $L_{H}=K L_{c}$. Namely this scale can be associated with the effective length in the Theory of Critical Distance in the framework of elasto-plastic models.

\section{FATIGUE LENGTH SCALES. INTERPRETATION OF BATHIAS-PARIS'S DIAGRAM OF FATIGUE CRACK GROWTH}

A pplication of the TCD to fatigue problems assumes that fatigue damage depends on the stress field distribution in the vicinity of the stress concentrator and the appropriate stress field parameter responsible for the damage in fatigue process zone could be introduced. It was proposed the calculation of the effective stress field parameter in the process zone according to the following relationship [27]:

$$
\Delta \sigma_{e f f}=\frac{1}{L_{e f f}} \int_{0}^{L_{e f f}} \Delta \sigma_{1}(\theta=0, r) \cdot[1-\chi \cdot r] d r=\Delta \sigma_{0},
$$

where $\chi$ is the stress gradient of the elasto-plastic stress field:

$$
\chi=\frac{1}{\sigma_{1}(\theta=0, r)} \cdot \frac{d \sigma_{1}(\theta=0, r)}{d r}
$$


Eq. (9) defines the effective stress using a weighted average of the elasto-plastic stress field damaging the fatigue process zone over a line having length equal to $L_{e f f}$. It is evident that the length $L_{e f f}$, as the integration path, is assumed to be dependent on the notch geometry. The non-locality effect is the key structural factor responsible for the characteristic length $L_{e f f}$ providing the correct predictions for the application of the TCD [28].

There is data concerning the TCD application in predicting the high-cycle fatigue strength of real mechanical components. The $L_{e f f}$ value in fatigue damage is associated somehow with the grain size, but, in general, its value is of an order of magnitude higher than the average grain size. This experimental observation in the fatigue process zone leads to the definition of structural volume concept for the interpretation of link between the linear-elastic stress distribution within such a volume and the initial crack path. The structural volume idea takes as starting point for the assumption that all physical processes resulting in the formation of fatigue cracks are confined within a finite volume. The size of this volume is assumed to be constant (but different for different materials) and it depends on the stress field damaging the fatigue process zone. Mentioned features of fatigue damage and fatigue crack advance in the interpretation of the TCD can be explained in terms of singularity dualities. The illustration of both these approaches (the TCD and duality of singularities) can be given by the interpretation of the Bathias-Paris diagram of fatigue crack growth, when both, stress and stressintensity based scenario of damage-failure transition, are presented.

The solutions (4) and (8) represent two types of singularities (two attractors corresponding to intermediate asymptotic solutions for stress distribution and damage kinetics), which provide the interpretation of the Bathias-Paris diagram of fatigue crack initiation and growth, Fig.4. The blow-up damage kinetics is the consequence of specific nonlinearity (metastability) of free energy release in the presence of non-locality effects of defects interaction that allows the explanation of transition from damage localization to the fatigue crack nucleation stage.

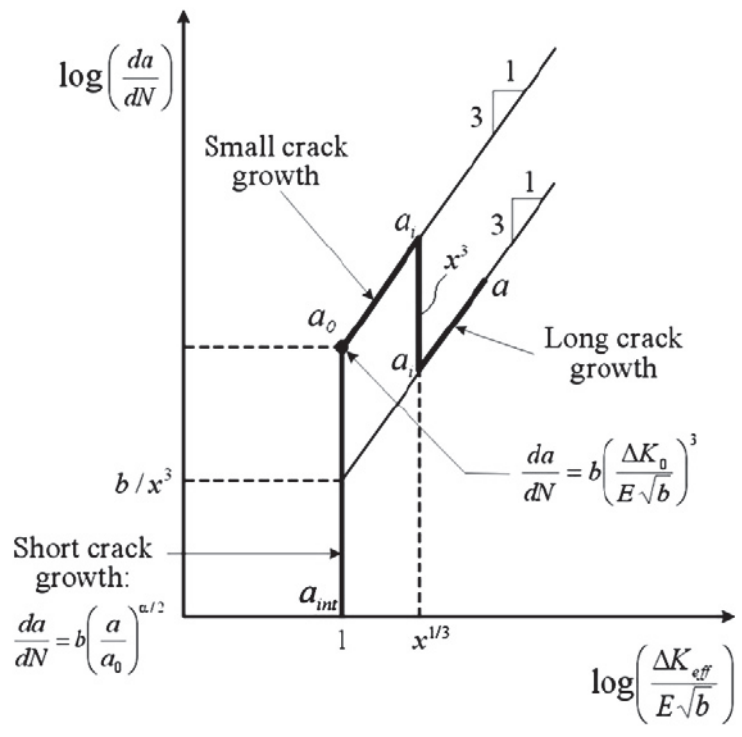

Figure 4. Crack advance diagram in HCF [29]: is the Burgers vector, $\Delta K_{0}$ and $\Delta K_{e f f}$ are stress intensity factors corresponding to the crack lengths $a_{0}$ and $a_{i}$.

The $\Delta K$ - independent area of fatigue crack initiation corresponds to the transition to the blow-up damage kinetics with explosive jump from structure dependent scales of defects $a_{\text {int }}$ to macroscopically recognized $a_{0}$. The power law $d a / d N=b\left(a / a_{\text {int }}\right)^{n / 2}$ reflects the self-similar (blow-up) stage of damage kinetics over $L_{c}$ scale that allows to estimate $a_{0} \sim L_{c}$. This length is related to the stress based scenario of fatigue crack nucleation.

Starting from this scales $a_{0}$ the singularity related to the stress intensity factor $\Delta K_{0}$ is combined with the blow-up singularity (6), that provides fatigue crack kinetics up to the scale $a_{l}$. The drop of the crack velocity is the consequence of subjection of crack kinetics to the stress intensity factor $\Delta K_{t h}$ according to the Paris law. The process zone scale in this 
case is associated with the $L_{H}$ length. This analysis allows the natural conclusion that variety of crack paths and characteristic lengths are related to duality of singularities caused by asymptotic self-similar solution for stress distribution at the crack tip and intermediate singular (blow-up) damage kinetics in the process zone.

Mentioned scenario can be illustrated by the morphological images of crack nucleation and propagation in the conditions of Very High Cycle fatigue, Fig.5 [29].

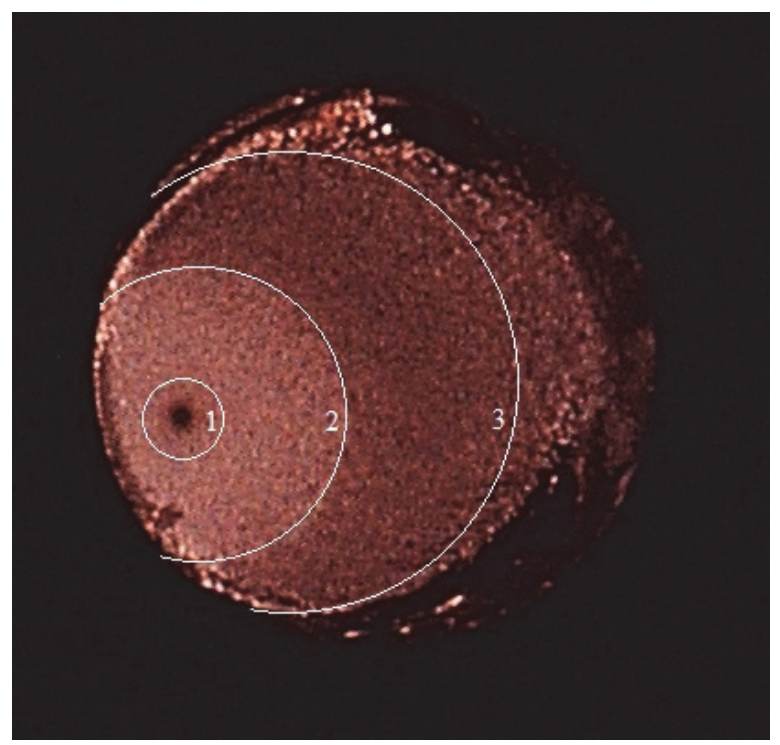

Figure 5. Fracture surface of Ti6Al4V alloy in Very High Cycle fatigue regime with the images of characteristic areas of fracture surface [30].

Different mechanisms related to above mentioned scenario were supported studing the scaling (spatial invariance) properties of characteristic area of fracture surface rughness. The surface roughness was analysed by nterferometerprofiler New View 5010 to establish quantitative characteristics of the fracture surface in terms of the scaling Hurst exponent. Three distinct zones with strongly different exponent were observed (Fig. 5): the area 1 of the size $\sim 100-300$ $\mu \mathrm{m}$ corresponds to the zone of damage localization (associated with $L_{c}$ ); the area 2 representing the image of crack advance in the presence of two singularities, given by the solutions (4) and (8), with the following re-subjection of crack kinetics (area 3) to the stress intensity fator.

\section{DiscussioN}

he existence of two singularities related to the stress field at the crack tip and blow-up kinetics of damage localization is considered as the physical basis for the interpretation of the Theory of Critical Distances. The free energy metastability of solid with defects and corresponding free energy release explain the conception of the Finite Fracture Mechanics in the presence of the finite amplitude energy barrier. The variety of crack paths is analyzed as duality of inherently linked two types of singularities related to the singularity of multiscale damage kinetics under crack nucleation and singularity of stress field at the crack tip as the classical framework of fracture mechanics. The singularity of multiscale damage kinetics is a natural precursor of crack nucleation that could provide in some cases the totally independent scenario of fracture from the stress singularity at the crack tips. The influence of two singularities with the nature of intermediate asymptotical solutions for stress at the crack tip and damage localization kinetics over the set of spatial scales represents two attractors, which provides the variety of crack paths for corresponding loading conditions and the existence of characteristic scales that could be associated with the critical distance lengths. 


\section{ACKNOWLEDGEMENTS}

uthor thanks Prof. Valery Matveyenko for the proposal to present the paper in the issue.
Research was supported by the Russian Foundation of Basic Research (project n. 17-01-00687a).

\section{REFERENCES}

[1] Taylor, D. (2008). The theory of critical distances, Engng, Fract. Mech., 75, pp. 1696-1705.

[2] Susmel, L., Taylor, D. (2008). On the use of the Theory of Critical Distances to predict static failures in ductile metallic materials containing different geometrical features, Engng. Fract. Mech.,75, pp. 4410-4421.

[3] Novozhilov, V.V. (1969). On a necessary and sufficient criterion for brittle strength, Prik. Mat. Mek., 33, pp.201210.

[4] Whitney, J.M, Nuismer, R.J. (1974). Stress fracture criteria for laminated composites containing stress concentrations, J, Compos. Mater. 8, pp. 253-265.

[5] Taylor, D. (2004). Predicting the fracture strength of ceramic materials using the theory of critical distances, Engng. Fract. Mech., 71, pp. 2407-2416.

[6] Taylor, D, Wang, G. (2000). The validation of some methods of notch fatigue analysis. Fatigue Fract. Engng. Mater. Struct., 23, pp.387-94.

[7] El Haddad, M.H., Topper, T.H., Smith, K.N. (1979). Prediction of non propagating cracks. Engng. Fract. Mech., 11, pp.573-84. Susmel, L. (2008). The theory of critical distances: a review of its applications in fatigue. Engng. Fract. Mech., 75, pp. 1706-1724.

[8] Taylor, D., Cornetti, P., Pugno, N. (2005). The fracture mechanics of finite crack extension. Engng. Fract. Mech., 72, pp. 1021-38.

[9] Taylor, D, Cornetti, P. (2005). Finite fracture mechanics and the theory of critical distances. In: Aliabadi M.H., editor. Advances in Fracture and Damage Mechanics IV. EC, Eastleigh UK, pp. 565-70.

[10] Hashin, Z. (1996). Finite thermoelastic fracture criterion with application to laminate cracking analysis. J. Mech. Phys. Solids, 44. pp. 1129-45.

[11] Leguillon, D. (2002). Strength or toughness? A criterion for crack onset at a notch. Eur J Mech A/Solids, 21, pp.6172.

[12] Cornetti, P., Pugno, N, Carpinteri A., Taylor D. (2006). Finite fracture mechanics: a coupled stress and energy criterion. Engng Fract Mech., 73, pp. 2021-2033.

[13] Taylor, D., Bologna, P., Bel Knani, K. (2000). Prediction of fatigue failure location on a component using a critical distance method. Int. J. Fatigue, 22, pp. 735-742.

[14] Griffith, A.A. (1921) The phenomena of rupture and flow in solids. Phil. Trans. Roy. Soc. London, Ser. A. 221, pp. 163-198.

[15] Irwin, G.R., (1957). Analysis of stresses and strains near the end of a crack traversing a plate. Journal of Applied Mechanics, 24, pp. 361-364.

[16] Barenblatt, G.I. (1962). The mathematical theory of equilibrium cracks in brittle fracture. Advances in Applied Mechanics, 7, pp. $55-129$.

[17] Fraenkel, Ya..I. (1952). Theory of reversible and non-reversible cracks in solid. Journal of Technical Physics, 22, pp. 1857-1866.

[18] Naimark, O.B. (2000). Collective behavior of cracks and defects (plenary lecture) In : D Miannay, P.Costa, D. Francois, A. Pineau, eds. Advances in Mechanical Behavior, Plasticity and Damage. Elsevier, 1, pp.15-28.

[19] Naimark, O.B. (2004). Defect Induced Transitions as Mechanisms of Plasticity and Failure (plenary lecture). In: G. Capriz and P. Mariano, eds. Advances in Multifield Theories of Continua with Substructure, Birkhäuser, Boston, pp.75-114.

[20] Beljaev, V.V., Naimark, O.B. (1990). Kinetics of multi-hotspot failure under shock wave loading Sov. Phys. Doklady, 312, pp.289-293.

[21] Bellendir, E.N., Beljaev, V.V., Naimark, O.B. (1989). Kinetics of multi-hotspot failure in the spall conditions. JETPh Letters, 15, pp. 90-93. 
[22] Naimark, O.B. (2016). Energy release rate and criticality of multiscale defects kinetics. Int. J. Fracture, 202, pp. 271 279.

[23] Naimark, O.B., and Uvarov, S.V. (2004). Nonlinear crack dynamics and scaling aspects of fracture (experimental and theoretical study), Int. J. Fracture, 128, pp. 285-292.

[24] Naimark, O.B., Uvarov, S.V., Davydova, M.M., Bannikova, I.A. (2017). Multiscale statistical laws of dynamic fragmentation. Physical Mesomechanics, 20, pp. 90-101. DOI: 10.1134/S1029959917010088.

[25] Kurdyumov, S.P. (1988). Evolution and self-organization laws of complex systems. International Journal of Modern Physics, 1, pp. 299-327.

[26] Qylafku, G., Azari, Z., Kadi, N., Gjonaj, M., Pluvinage, G. (1999). Application of a new model proposal for fatigue life prediction on notches and key-seats. Int J Fatigue, 21, pp.753-60.

[27] Atzori, B., Meneghetti, G., Susmel, L. (2005). Material fatigue properties for assessing mechanical components weakened by notches and defects. Fract. Engng. Mater. Struct., 28, pp. 83-97.

[28] Huang, Z., Wagner, D., Bathias, C., Paris, P.C. (2010). Subsurface crack initiation and propagation mechanisms in gigacycle fatigue, Acta Materialia, 58, pp. 6046-6054.

[29] Betekhtin, V.I., Kadomtsev, A.G., Narykova, M.V., Bannikov, M.V., Naimark, O.B., Abaimov, S.G., Akhatov, I.S., Palin-Luc, T. (2017). Experimental and theoretical study of multiscale damage-failure transition in very high cycle fatigue, Physical Mesomechanics, 20, 78-89. 